\title{
Biocidal spectrum of a rice field cyanobacterium Nostoc sp.
}

\author{
*P. Jaiswal ${ }^{1^{*}}$, R. Prasanna ${ }^{2}$, P.K. Singh ${ }^{3}$ \& Prem Dureja ${ }^{4}$ \\ ${ }^{1}$ Centre for Post Harvest Engineering and Technology, Ludhiana-141004 \\ 2 Division of Microbiology, Indian Agricultural Research Institute (IARI), New Delhi 110012, India, \\ ${ }^{3}$ Department of Botany, Banaras Hindu University, Varanasi-221005, India \\ ${ }^{4}$ Division of Agricultural Chemicals, IARI, New Delhi-110012
}

\begin{abstract}
The antimicrobial efficacy of hexane, dichloromethane and ethyl acetate extracts of a rice-field cyanobacterium, Nostoc sp., were evaluated against cyanobacteria and phytopathogenic fungi. The maximum production of biocidal compounds was observed in cultures grown for 20 days under optimized conditions (phosphate $=1.4 \mathrm{mg} / \mathrm{l}$; light intensity $\approx 3000-4000 \mathrm{lux}$ ). Fractionation of the extracts showed six spots on silica-gel-coated plates. Hexane extract was the most potent biocide, showing marked (35-42\%) reduction in the growth of fungi, but no negative influences on seed germination or seedling growth of wheat, rice and mung bean, emphasizing its suitability for use in agriculture.
\end{abstract}

Keywords: Antifungal; extract; inhibition zone; metabolite; Pythium debaryanum; Rhizoctonia solani.

\section{Introduction}

Cyanobacteria constitute a heterogeneous group of phototrophic prokaryotes with characteristics in common to both bacteria and algae. They are often referred to as 'biofactories' due to their unique biosynthetic potential to produce wide range of compounds e.g enzyme, hormones, pigments, proteins, fatty acids/lipids, etc (Prasanna et al. 2010). They are distributed in a wide range of aquatic and terrestrial habitats owing to their high adaptive ability under diverse environmental conditions. Some species of cyanobacteria can proliferate in aquatic environments, leading to a significant deterioration of water quality- a phenomenon known as "water bloom". These water blooms are of tremendous biological significance because they have proved excellent sources of exciting molecules with immunomodulatory, bioregulatory and therapeutic potential (Namikoshi \& Rinehart 1996, Prasanna \& Jaiswal 2006, Jaiswal et al. 2007). However, increasing evidence indicates that besides these bloom forming strains, cyanobacteria colonizing other habitats may also produce wide variety of low molecular weight secondary metabolites with biological activities (Ghasemi et al. 2003; Jaiswal et al. 2005; Jaiswal et al. 2008; Prasanna et al. 2010).

The range of bioactivities shown by cyanobacterial compounds is very diverse and include antimicrobial, antiviral, immuno-modulatory, insecticidal and antioxidant activities, which can be agriculturally useful. They also serve as allelochemicals, providing a competitive advantage to the producer and contributing to the dominance of a strain in a particular habitat. A number of compounds have been isolated that exhibit biocidal activity against specific agriculturally important pathogens (fungi, nematodes) (Pushparaj et al. 2000, Nagle \& Wedges 2002, Volk \& Fulkert 2006, Prasanna et al. 2009, Chaudhary et al. 2010, Manjunath et al. 2010). Therefore cyanobacteria offer an effective soil-friendly alternative to agrochemicals for controlling plant pathogens while maintaining soil fertility and environmental safety. Cyanobacteria from local habitats can be a good source of potential biologically active substances responsible for the controlling the growth of local pathogenic microorganisms (Dukare et al. 2011). With this in mind, the current investigation was aimed at evaluating the biocidal spectrum of a rice-field cyanobacterium, Nostoc sp., and its potential as a biocontrol agent.

\footnotetext{
* Author for correspondence: Tel: +91-161-231-3170 Fax: +91-161-230-8670 email : pranitajaiswal@gmail.com
} 


\section{Materials \& Methods}

Nostoc sp. used in this investigation was isolated from a rice field grown at the Indian Agricultural Research Institute (Jaiswal et al. 2005). Two unicellular cyanobacterial strains (Synechococcus sp. and Synechocystis sp.) were used as test organisms. All these strains were grown and maintained in BG11 medium and incubated at $27 \pm 1{ }^{\circ} \mathrm{C}, 3000$ lux light intensity and 16:8 L:D cycles. The fungal strains (Pythium debaryanum, Fusarium oxysporum, Rhizoctonia solani, Rhizoctonia bataticola and Alternaria solani) used for evaluating fungicidal activity were obtained from the Division of Plant Pathology, IARI, New Delhi. The cultures were maintained on potato dextrose agar medium. The fungal strains were grown and maintained at $28 \pm 2{ }^{\circ} \mathrm{C}$ in a BOD incubator.

In order to study the effect of different physicochemical factors on algicidal activity, the basal medium was supplemented with different levels of $\mathrm{P}$ (half and twice the levels of the BG-11 medium). The effect of light intensities (1500-6000 lux) and temperature $\left(20-40{ }^{\circ} \mathrm{C}\right)$ was also evaluated.

The axenized isolate of Nostoc sp. was set for mass multiplication in 3L flasks and incubated under optimized conditions for maximum biocidal acticvity. The cultures were harvested in the exponential phase of growth by pelleting at high speed $(8000 \mathrm{rpm})$ for 20 minutes. The cell free extracts were pooled for the extraction of secondary metabolites.

The culture filtrates were partitioned with non-polar (n-hexane), polar (dichloromethane) and highly polar (ethyl acetate) solvents sequentially to extract all the compounds present in culture filtrates. The organic phase was then separated from the aqueous phase and dried over anhydrous sodium sulphate. The extracts were then subjected to rotary evaporation under pressure. The solid extract was dissolved in acetone and stored at $4{ }^{\circ} \mathrm{C}$ until further analyses.

The bioactivity of the cyanobacterial filtrate, crude extracts and the partially purified compounds were tested on a lawn of two unicellular cyanobacteria (Synechococcus sp. and Synechocystis sp.) and five phytopathogenic fungi by the disc-diffusion technique (Jaiswal et al. 2005). The diameter of the inhibition zone was taken as the measure of toxicity. The percent inhibition of the fungal strain was also calculated by the food-poisoning technique and measuring the colony size of the fungal strain.

The inhibitory effect of the concentrated extracts was examined in agar-based media using 96-well ELISA plates based on the methodology developed by Prasanna et al. (2006). After rotary evaporation, extracts were weighed and dissolved in the desired volume of acetone and a series of different dilutions prepared. Five replicates of each concentration were placed in sterilized medium (BG-11 and potato dextrose agar) in the wells of the ELISA plates. Each well was inoculated with the test organism (cyanobacterium/fungus) and incubated under optimal growth conditions along with suitable controls.

The effect of culture filtrate/crude extract of the cyanobacterium was tested on seed germination, length of coleoptile and radicle of wheat (var HD2689), rice (var Pusa Basmati 1) and mung bean (var Pusa Ratna). Seeds were surface-sterilized using $0.1 \%$ mercuric chloride and washed thoroughly with sterile water. They were imbibed overnight in sterile water in the dark at room temperature and kept for germination on water agar (2\% agar in distilled water containing minimum inhibitory concentration levels of cyanobacterial extracts). The plates were incubated in the dark at $25 \pm 2{ }^{\circ} \mathrm{C}$. The percentage germination of the seeds was calculated after $24 \mathrm{~h}$ of incubation, and the length of the coleoptile and radicle measured after 48 h (Jaiswal et al. 2010).

Thin-layer chromatography of the samples separated the compounds present in the Nostoc extracts (Jaiswal et al. 2010). Silica-gel-coated plates $(0.15 \mathrm{~mm}$ thick) activated in a hot air oven at $120{ }^{\circ} \mathrm{C}$ were used. The crude extract dissolved in acetone was spotted on the TLC 
plates and then passed through different solvent systems to achieve maximum separation of the constituent compounds.

Statistical analysis was carried out using Microsoft Excel. At least three independent experiments were conducted and readings were taken in triplicate for each.

\section{Results}

The culture filtrate of Nostoc showed algicidal activity (Jaiswal et al. 2005), which was significantly affected by culture age. Time-course studies indicated that 5-d-old cultures (early exponential phase) exhibited very little algicidal activity, but gradual enhancement was observed with increasing culture age (Figure 1A). The maximum algicidal activity was recorded with 20-d-old cultures, which were 3.8- (Synechococcus) and 2-fold (Synechocystis) higher than 5-d-old culture. Further ageing (to 25 days) led to a slight decline in biocidal activity.
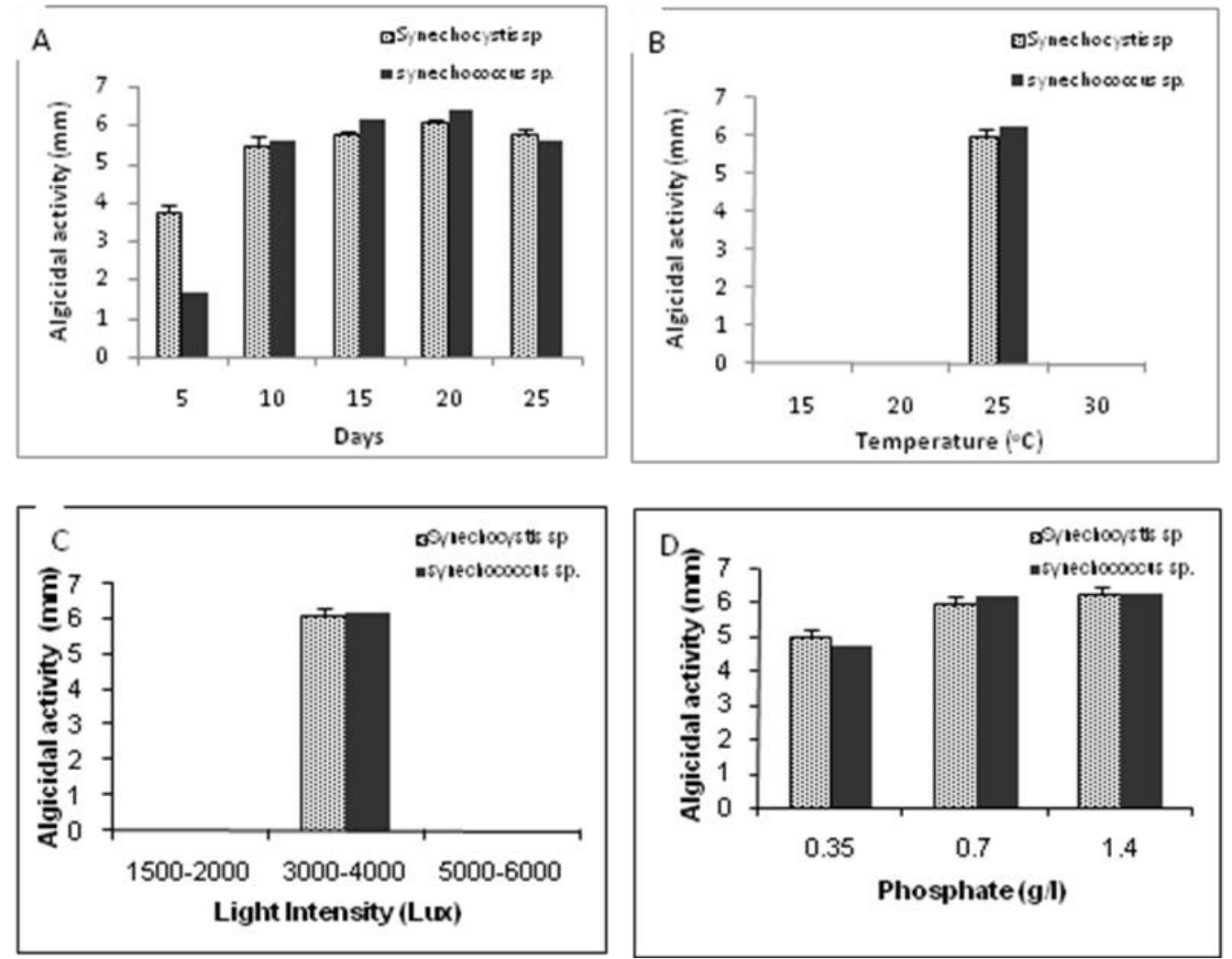

Figure 1: Effect of incubation (A), temperature (B), light intensity (C) and phosphate levels (D) on the algicidal activity of Nostoc sp. (measured as diameter of the inhibition zone).

A shift in the temperature from the optimal range $\left(25 \pm 2{ }^{\circ} \mathrm{C}\right)$ resulted in complete loss of biocidal activity (Figure 1B). Similarly, a change in light intensity from the optimal level (3000- 4000 lux) also resulted in loss of algicidal activity (Figure 1C). The highest algicidal values were recorded when the phosphate concentration was doubled $(1.4 \mathrm{~g} / \mathrm{l})$ as compared to basal level in BG-11 medium, but there were significant differences between the two test organisms. The algicidal activity at lower levels of $\mathrm{P}(0.35 \mathrm{~g} / \mathrm{l})$ was substantially reduced (Figure 1D).

The addition of culture filtrate @ 10\% (v/v) of Nostoc sp. led to a 1.5- (Synechocystis) and 1.2-fold (Synechococcus) lowering of protein content on the $2^{\text {nd }}$ day of incubation (Figure 
$2 \mathrm{~A})$. It reduced much further by the end of $8^{\text {th }}$ day. The chlorophyll content was also strongly affected by the culture filtrate of Nostoc sp. (Figure 2B), 4.9- (Synechocystis) and 6.9-fold (Synechococcus) lower than the control on the 8th day of incubation.
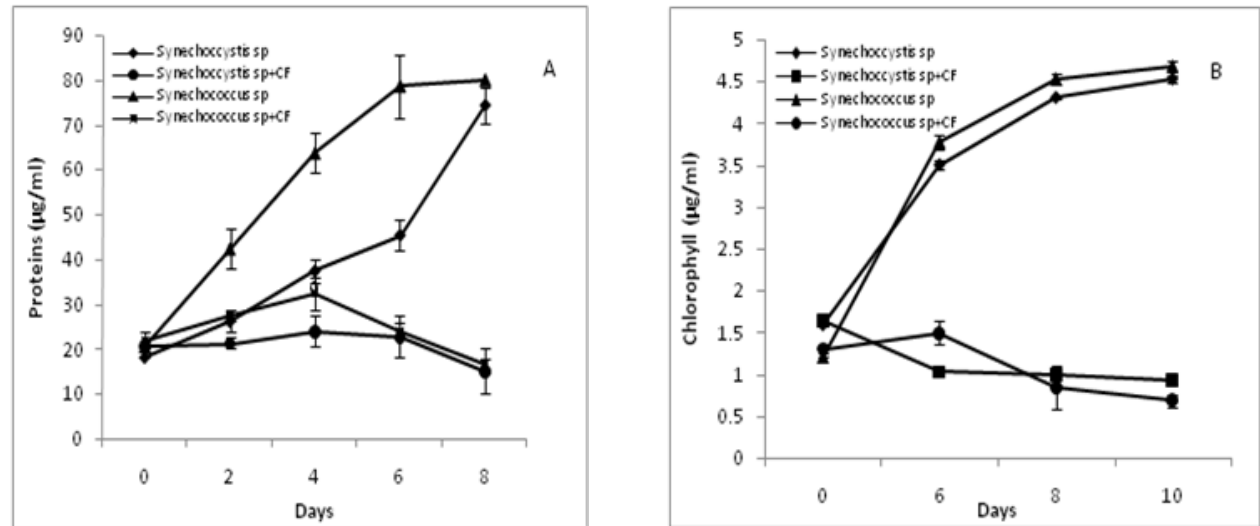

Figure 2: Effect of culture filtrate $(\mathrm{CF})$ of Nostoc sp. on protein (A) and chlorophyll (B) content of Synechococcus and Synechocystis sp.

The crude extracts of cyanobacteria (grown under optimized conditions) using nhexane, dichloromethane and ethyl acetate were tested for biocidal activity on the two unicellular cyanobacteria and five phytopathogenic fungi. Biocidal activity was recorded in all the extracts with varied sensitivity (Figure 3). The hexane extract had maximum biocidal (algicidal and fungicidal) activity in terms of the zone of inhibition. Differences in algicidal activity by the dichloromethane and ethyl acetate extracts were not significant, but the former was observed to be more potent in showing fungicidal activity.

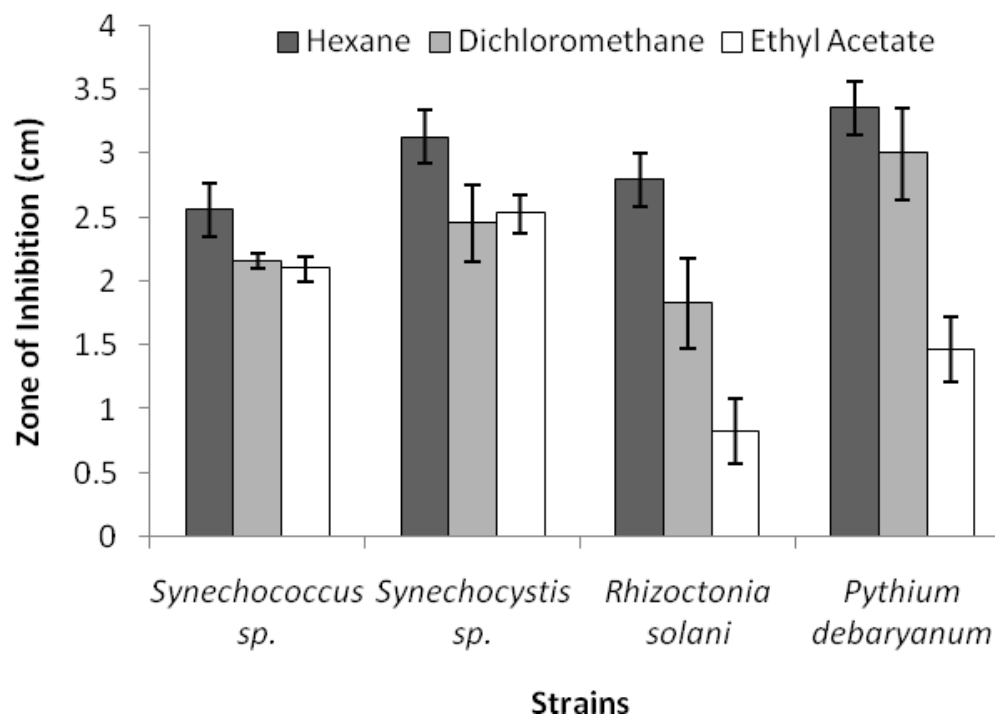

Figure 3: Biocidal activity (measured as zone of inhibition) of crude extract of Nostoc sp. against two unicellular cyanobacteria (Synechococcus and Synechocystis sp.) and two phytopathogenic fungi ( $R$. solani and $P$. debaryanum).

The crude extract also showed significant reductions in growth of the cyanobacterial and fungal strains (Table 1). The maximum inhibition was recorded with the hexane extract. Among the two fungal strains, $P$. debaryanum exhibited a $42 \%$ reduction, while among the two cyanobacterial strains Synechocystis sp. was found to be more sensitive to the extract. 


\begin{tabular}{lrrr}
\hline Test organism & \multicolumn{3}{c}{ Reduction in growth $(\%)$} \\
& n-hexane & dichloromethane & ethyl acetate \\
\hline Synechococcus sp. & 32.0 & 27.0 & 26.3 \\
Synechocystis sp. & 39.1 & 30.8 & 31.6 \\
Rhizoctonia solani & 35.0 & 22.8 & 10.3 \\
Pythium debaryanum & 42.0 & 37.5 & 18.4 \\
\hline
\end{tabular}

Table 1: Effect of cyanobacterial extract (Nostoc sp.) on the percent reduction in growth of test organisms.

The minimum inhibitory concentration required for the algicidal and fungicidal activity was calculated by serial dilution of the concentrated extract: for algicidal activity this was found to be $31.2 \mathrm{ppm}$, while it was $62.5 \mathrm{ppm}$ for fungicidal activity.

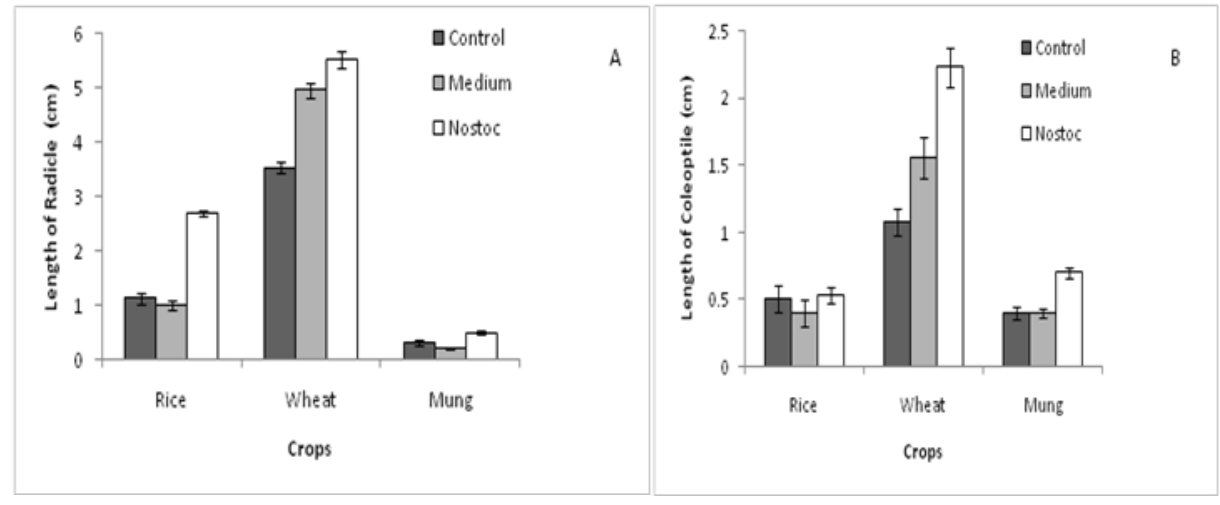

Figure 4: Effect of cyanobacterial culture filtrate on length of the radicle (A) and coleoptile (B) of rice, wheat and mung seedlings.

The effect of the culture filtrate/extract on important crop plants was studied. Two concentrations of the extract were used $-31.25 \mathrm{ppm}$ and $62.5 \mathrm{ppm}$ (based on the minimum inhibitory concentration). The lower concentration had no effects on germination of wheat, mung and rice seeds (data not shown). The culture filtrate enhanced radicle and coleoptile lengths for all three crop seedlings (Figure 4). The extracts had no negative influences, except slight reduction in lengths for mung bean seedlings at the $62.5 \mathrm{ppm}$ concentration (Table 2).

\begin{tabular}{lcccccc}
\hline Treatments & \multicolumn{2}{c}{} & \multicolumn{2}{c}{ length $(\mathrm{cm})$} & \multicolumn{2}{c}{ mung bean } \\
& coleoptile & radicle & coleoptile & radicle & coleoptile & radicle \\
\hline & & & & & & \\
Control (water) & $1.08 \pm 0.05$ & $3.53 \pm 0.58$ & $0.50 \pm 0.02$ & $1.13 \pm 0.10$ & $0.40 \pm 0.10$ & $0.30 \pm 0.06$ \\
Control (acetone) & $1.04 \pm 0.03$ & $3.53 \pm 0.36$ & $0.50 \pm 0.05$ & $1.12 \pm 0.00$ & $0.40 \pm 0.00$ & $0.30 \pm 0.10$ \\
$31.25 \mathrm{ppm}$ & $1.10 \pm 0.07$ & $3.70 \pm 0.06$ & $0.50 \pm 0.08$ & $1.15 \pm 0.10$ & $0.40 \pm 0.10$ & $0.30 \pm 0.10$ \\
$62.50 \mathrm{ppm}$ & $1.08 \pm 0.10$ & $3.65 \pm 0.20$ & $0.50 \pm 0.02$ & $1.15 \pm 0.03$ & $0.36 \pm 0.03$ & $0.28 \pm 0.11$ \\
\hline
\end{tabular}

Table 2: Effect of different concentrations of cyanobacterial extract on the length of coleoptile and radicle of selected seedlings.

Thin-layer chromatography fractionation of crude cyanobacterial extract showed the presence of 2 (n-hexane), 5 (dichloromethane) and 3 (ethyl acetate) spots (Table 3) when nhexane: benzene (1:1) was used as the solvent system. Fraction A with $\mathrm{Rf}$ value 0.07 was common to all solvents, while fraction B was common to hexane and dichloromethane, and 
fraction $\mathrm{C}$ to dichloromethane and ethyl acetate solvents. Fractions D (ethyl acetate), E and $\mathrm{F}$ (dichloromethane) were unique to single solvents.

\begin{tabular}{llrrrrrr}
\hline Solvent & bands & \multicolumn{7}{c}{ Rf values } \\
& & A & B & C & D & E & F \\
n-Hexane & 2 & 0.07 & 0.16 & - & - & - & - \\
Dichloromethane & 5 & 0.07 & 0.16 & 0.22 & - & 0.60 & 0.94 \\
Ethyl acetate & 3 & 0.07 & - & 0.22 & 0.29 & - & - \\
\hline
\end{tabular}

Table 3: Comparative rate of flow of different fractions of crude extract of Nostoc sp. using thin layer chromatography.

The extracted metabolite (n-hexane and dichloromethane extracts) was partially purified using preparatory thin layer chromatography and fraction A was found to exhibit the maximum algicidal activity, followed by fractions B and E; maximum fungicidal activity was recorded for fraction $\mathrm{B}$ followed by fraction A. Other fractions did not show any fungicidal activity.

\section{Discussion}

Cyanobacteria, a large group of structurally diverse, ecologically adaptive, Gram negative prokaryotes, have long been known for their potential use in agriculture as biofertilizer, soil conditioner and plant growth regulation due to their ability to secrete a number of compounds such as polysaccharides, peptides, etc, during growth. They have recently drawn the attention of biologists worldwide for their rich source of novel bioactive compounds with multifaceted applications (e.g. in agriculture, industry, pharmaceuticals). Studies have revealed that secondary metabolite production in cyanobacteria is significantly influenced by culture age. Most of the reports indicate that it is released into the medium in the late exponential phase after cell lysis (Ray \& Bagchi 2001, Dias et al. 2002). In the current investigation with Nostoc sp., algicidal activity was detected, on the lawn of two unicellular cyanobacteria (Synechococcus and Synechocystis sp.) at all the stages of growth, increasing with time and with a maximum in the stationary phase culture. The correlation between culture age and activity is similar to earlier reports (Ray \& Bagchi 2001, Dias et al. 2002). Volk (2007) also reported that the content of exo-metabolites of a cyanobacterium Nostoc insulare changes with growth phase; during the linear growth phase, a non-toxic metabolite was prevalent, while in the stationary phase the content of antimicrobial cytotoxic metabolite was enhanced.

The cyanobacterium in the current investigation showed loss of algicidal activity when grown in culture conditions (i.e light and temperature) other than the optimal range. There have been contradictory reports regarding the influence of light and temperature on the production of biocidal compounds. A few reports suggest that an increase in light intensity has a positive effect on toxin production (Utkilin \& Gjolme 1992), while some others suggest that there is no significant effect (Wicks \& Thiel 1990). Most of the earlier reports also suggest that toxin production by cyanobacteria is less at the upper and lower limits of the temperature optima for the species (Ressom et al. 1994, Jaiswal et al. 2007, Jaiswal et al. 2008), which agrees with the results obtained in the current investigation.

In order to study the effect of algicidal compound on the test organism, the protein and chlorophyll content of two unicellular cyanobacteria were estimated. The addition of culture filtrate brought about a significant reduction in the protein and chlorophyll content by the $2^{\text {nd }}$ day of incubation, indicating that the metabolite may affect some of the primary metabolic processes. Bagchi \& Ray (2001) also reported that the cyanobacterial extract of Oscillatoria sp. showed PSII-inhibiting activity, besides algicidal activity. 
In order to understand the polar/nonpolar nature of the biocidal metabolite, the culture filtrate was partitioned using different solvents. Maximum activity was observed in hexane extract, indicating the non-polar nature of the active metabolite. TLC fractionation of the extracts resolved into a number of spots, indicating the presence of more than one compound in each extract, further confirmed by presence of a number of peaks in the GC analyses. Among the various fractions, algicidal activity was shown by fraction no. A, B and $\mathrm{E}$ in descending order of activity. The presence of a large number of metabolites with varying toxicity levels and host range has been reported in cyanobacteria (Jaki et al. 2000, Volk \& Mundt 2007, Jaiswal et al. 2010).

The effect of cyanobacterial filtrate and crude extract on seed germination and growth of important crop plants revealed the positive influence of culture filtrate on seedling growth, which may be due to the presence of some growth-promoting substances in the cyanobacterial extract. Though we have not tested the cyanobacterial extract for the presence of amino acids, lipids or indole acetic acid, such biomolecules are not uncommon in cyanobacterial extracts (Kartikeyan et al. 2009). More importantly, the crude extract of the culture filtrate did not have any negative influence on seed germination and growth of seedlings, an important advantage for the potential use of extract as a fungicidal agent, because non-target biota are not affected.

Cyanobacteria have long been known for their potential for $\mathrm{N}_{2}$ fixation in rice fields, and hence have been traditionally used as biofertilizer, with only few reports on the production of antimicrobial substances from rice-field cyanobacteria (Ghasemi et al. 2003). The rice rhizosphere is known to be the site of a large number of microorganisms belonging to diverse groups including cyanobacteria (Prasanna et al. 2009). The presence of biologically active substances may serve as a defence strategy against other co-occurring organisms such as bacteria, fungi and viruses. The current study highlights the potential of these cyanobacteria as biocontrol agents against various economically important plant pathogens, thus enhancing their value as inoculants.

\section{Acknowledgements}

The authors thank the Council of Scientific \& Industrial Research (CSIR), and the Indian Council of Agricultural Research, New Delhi, India for providing financial assistance in the form of projects. We gratefully acknowledge the provision of the facilities required for carrying out this investigation by the Division of Microbiology, Division of Agricultural Chemicals AS \& EC Division, CIPHET, Ludhiana.

\section{References}

Bagchi SN \& Ray S (2001) Extraction and purification of an algicidal metabolite from a cyanobacterium Oscillatoria latevirens. Indian Journal of Microbiology 41: 163-167

Chaudhary V, Prasanna R, Gupta V, Singh SB, Natarajan C \& Nain L (2010) Development of microtitre-platebased assay for evaluation of fungicidal potential and microscopic analyses of cyanobacterial metabolites with fungal mycelia. Archives of Phytopathology \& Plant Protection 43: 1435-1444

Dias E, Pereira P \& Franca S (2002) Production of paralytic shellfish toxins by Aphanizomenon sp. LMECYA 31 (cyanobacteria). Journal of Phycology 38: 305-308

Dukare AS, Prasanna R, Dubey SC, Nain L, Chaudhary V, Singh R \& Saxena A (2011) Evaluating novel microbe-amended composts as biocontrol agents in tomato. Crop Protection 30 (4): 436-442

Ghasemi Y, Tabatabai Y, Shokravi S, Soltani N \& Zarrini G (2003) Antifungal and antibacterial activity of paddy-field cyanobacteria from the north of Iran. Journal of Sciences Islamic Republic of Iran 14: 203-209

Jaiswal P, Prasanna R \& Singh PK (2005) Isolation and screening of rice field cyanobacteria exhibiting algicidal activity. Asian Journal of Microbiology, Biotechnology \& Environmental Sciences 7: 367-373

Jaiswal P, Prasanna R \& Singh PK (2007) Factors influencing algicide production by Microcystis sp. and its effect on selected cyanobacteria. In Gupta RK \& Pandey VD (eds.). Advances in Applied Phycology. New Delhi (India): Daya Publishing House. 75-84

Jaiswal P, Prasanna R \& Singh PK (2011) Characterization of the biocidal spectrum of extracellular filtrates of Microcystis aeruginosa. Indian Journal of Microbiology 51: 509-514

Jaiswal P, Singh PK \& Prasanna R (2008) Cyanobacterial bioactive molecules: an overview of their toxic properties. Canadian Journal of Microbiology 54: 701-717 
Jaki B, Heilman J \& Sticher O (2000) New antibacterial metabolites from the cyanobacterium Nostoc commune (EAWAG 122b). Journal of Natural Products 63: 1283-1285

Karthikeyan N, Prasanna R, Sood A, Jaiswal P, Nayak S \& Kaushik BD (2009) Physiological characterization and electron microscopic investigations of cyanobacteria associated with wheat rhizosphere. Folia Microbiologica 54: 43-51

Manjunath M, Prasanna R, Lata, Dureja P, Singh R, Kumar A, Jaggi S \& Kaushik BD (2010) Biocontrol potential of cyanobacterial metabolites against damping-off disease caused by Pythium aphanidermatum in solanaceous vegetables. Archives of Phytopathology \& Plant Protection 43: 666-677

Nagle DG \& Wedge DE (2002) Antifungal properties of cyanobacteria and algae: ecological and agricultural implications. In Inderjit \& Malik AU (eds.). Chemical ecology of plants: allelopathy in aquatic and terrestrial ecosystems. Switzerland: Birkhauser. 7-32

Namikoshi M \& Rinehart KL (1996) Bioactive compounds produced by cyanobacteria. Journal of Industrial Microbiology \& Biotechnology 17: 373-384

Prasanna R, Gupta V, Natarajan C \& Chaudhary V (2009) Allele mining for chitosanases and microcystin-like compounds in Anabaena strains. World Journal of Microbiology \& Biotechnology 26(4): 717-724

Prasanna R \& Jaiswal P (2006) Algal Toxins: boon or bane to mankind. Science \& Culture 72: 9-10

Prasanna R, Jaiswal P, Nayak S, Sood A \& Kaushik BD (2009) Cyanobacterial diversity in the rhizosphere of rice and its ecological significance. Indian Journal of Microbiology 49: 89-97

Prasanna R, Saxena AK, Jaiswal P \& Nayak S (2006) Development of alternative support system for viable count of cyanobacteria by most probable number method. Folia Microbiologica 51: 455-458

Prasanna R, Sood A, Jaiswal P, Nayak S, Gupta V, Chaudhary V, Joshi M \& Natarajan C (2010) Rediscovering cyanobacteria as valuable sources of bioactive compounds. Applied Biochemistry \& Microbiology 46:133147

Pushparaj B, Pelos, E \& Caroppo S (2000) Effect of Nodularia harveyana biomass on the incidence of root-knot nematode (Meloidogyne incognita) in tomato. Journal of Applied Phycology. 12: 489-492

Ray S \& Bagchi SN (2001) Nutrients and pH regulate algicide accumulation in cultures of cyanobacterium Oscillatoria latevirens. New Phytologist 149: 455-460

Ressom R, Soong FS, Filzgerald J, Turezynowicz LEI, Saadi O, Roder D, Maynard T \& Falconer I (1994) Health effects of toxic cyanobacterial (Blue-green algae). National Health \& Medical Research Council, Australian Government Publishing Service, Canberra, Australia

Utkilen H \& Gjolme N (1992) Toxin production by Microcystis aeruginosa as a function of light in continuous cultures and its ecological significance. Applied \& Environmental Microbiology 58: 1321-1325

Volk RB (2007) Studies on culture age versus exometabolite production in batch cultures of the cyanobacterium Nostoc imsulare. Journal of Applied Phycology 19: 491-495

Volk, RB \& Furkert FH (2006) Anti-algal, antibacterial and antifungal activity of two metabolites produced by cyanobacteria during growth. Microbiological Research 161: 180-186

Volk RB \& Mundt S (2007) Cytotoxic and non-cytotoxic exometabolites of the cyanobacterium Nostoc insulare. Journal of Applied Phycology 19: 55-62

Wicks JR \& Thiel PG (1990) Environmental factors affecting the production of peptide toxins in floating scums of the cyanobacterium Microcystis aeruginosa in a hypertrophic African reservoir. Environmental Science \& Technology 24: 1413-1418

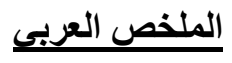

